\title{
The Tribond Bridged Bipyridine Complex-based In-fiber Interferometric Sensor design for $\mathrm{Cu}^{2+}$ Detection
}

\author{
Jiahui Huang ${ }^{1}$, Lijun $\mathrm{Li}^{1, *}$, Yumeng $\mathrm{Lv}^{1}$, Min $\mathrm{Li}^{1}$ \\ ${ }^{1}$ College of Information and Electrical Engineer, Shandong University of Science and Technology, Shandong Qingdao 266590, China
}

\begin{abstract}
We report a real-time, lightweight sensor based on in-fiber MZ interferometer coating bipyridine complex L[4-methyl-2,6-bis(pyridine-3-ylethynyl)aniline](L-ligand) in situ determination of free copper ion at micromolar levels. The sensor is made by fusing a section of thin-core fiber(TCF) in the middle of singlemode fiber(SMF). The L-ligand material is attached to the sensing surface of thin-core fiber to bind free copper ions in liquid environment with high affinity and selectivity. Investigation was carried out that the performance of the sensor is significantly improved with different length. The sensitivity of $\mathrm{Cu}^{2+}$ detection can reach $0.0573 \mathrm{~nm} / \mu \mathrm{M}$ (nanometer per micromolar) in linear range of $0 \mu \mathrm{M} \sim 25 \mu \mathrm{M}$ and the detection limit is $0.8726 \mu \mathrm{M}$. This is significantly lower than the allowable level of drinking water.
\end{abstract}

\section{Introduction}

Copper element is ubiquitously distributed in biological systems and natural environments, which has an important impact on the development and function of blood, central nervous system, immune system and viscera [1-2]. Thus it is regulated the person daily intake limit of $\mathrm{Cu}$ should be maintained at $2-5 \mathrm{mg}$, WTO rules of copper in drinking water limit threshold of $2 \mathrm{mg} / \mathrm{L}$, and US(EPA) standards for $1 \mathrm{mg} / \mathrm{L}[3]$. Hence the detection and analysis of copper ion concentration in the environment become essential.

In recent years, the reported methods for the detection of copper ions include spectral analysis (atomic absorption spectrometry, atomic emission spectrometry, fluorescence spectrometry)[4], spectrophotometry [5], electrochemical analysis [6], optical fiber sensing analysis [7]. These methods have many problems, such as long sample pre-treatment time, complex process and financial cost. In order to overcome this limitation, in our research work, we used a SMF-TCF-SMF sensitive fiber interference structure, and reported a method to measure the concentration of $\mathrm{Cu}^{2+}$ by coating a functional sensing surface with bipyridine complex L[4-methyl-2,6bis(pyridine-3-ylethynyl)aniline](L-ligand). The mechanism of optical fiber sensing is based on the external coupling of backward propagation cladding mode with environmental RI. Moreover, L-ligand can capture copper ions and change the refractive index around the sensor. By monitoring the change of infrared wavelength or the power change of spectral characteristics, a more sensitive, inexpensive and fast response time copper ions sensing scheme is proposed.

\section{Theory}

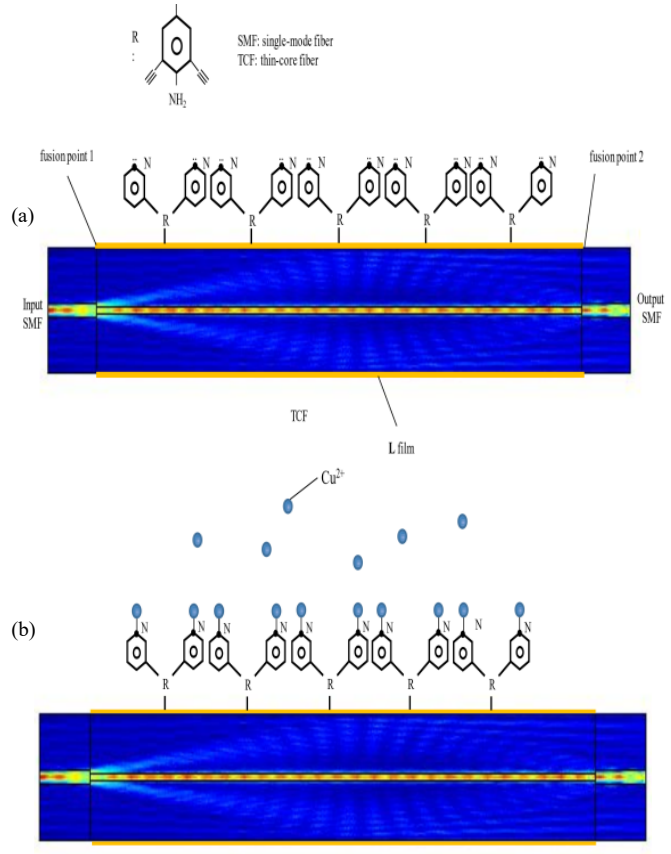

Fig. 1 structure diagram of MZ interferometric sensing based on L film (a) Schematic diagram of STS sensor structure and light transmission principle; (b) schematic diagram of copper ion sensing response

The sensor structure which is constructed by a sandwiching structure with a thin-core fiber spliced between two standard single mode fibers is shown in Fig.1. The wavelength of STS structure is formed by the interference of core mode and cladding mode[8]. When the external environment changes, the effective refractive index of the cladding mode will change, which cause the 
shift of the cladding mode central wavelength. At the same time, L-ligand is coated outside the cladding of thin-core diameter fiber with STS sensing structure. Due to the unsaturated covalent bond in the L-ligand, there are vacancy orbitals and flowing electron cloud in the Lligand, which interacts with copper ion to produce complex reaction[9]. Then the complex reaction changes the refractive index of coating surface material, resulting in the shift of MZ interference spectrum. By observing the drift in real time, the sensing of copper ion concentration is realized.

The relationship between the dip wavelength $\lambda_{D}$ and the coating film RI can be expressed as follows:

$$
\lambda_{D}=\left[\left(n_{\text {core }}-n_{\text {coating }}\right) l\right] / K
$$

among them, $n_{\text {core }}$ and $n_{\text {coating }}$ is the effective refractive index of the optical fiber core and the coating, $K$ is mode order and an integer, and $l$ is the length of the TCF.

\section{Sensing experiment and discussion}

\subsection{Sensing experiment device}

The experimental device of the sensor is shown in Fig 2.The fusion equipment used in the laboratory is FITELs177, and the spectrometer is YOKOGAWA-AQ6370. The TCF used in the laboratory is produced by YOFC. The mode field diameter of TCF at $1550 \mathrm{~nm}$ is about $4.5 \mu \mathrm{m}$, the SMF cladding diameter is $125 \mu \mathrm{m}$ and the core diameter is $9 \mu \mathrm{m}$.

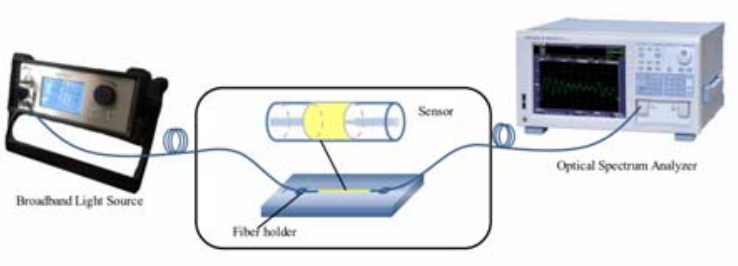

Fig. 2 Schematic diagram of in fiber copper ion sensing experimental device

\subsection{Detection of copper ion concentration with different length sensors}

In the experiment, in-fiber sensors with thin-core diameter of $3 \mathrm{~cm}, 5 \mathrm{~cm}$ and $7 \mathrm{~cm}$ were coated with L-film, which were respectively recorded as STS1, STS2 and STS3, and immersed in different concentrations of copper ion solution for wavelength monitoring. When the length of thin-core diameter optical fiber is $3 \mathrm{~cm}$, the response spectrum superposition diagram of the sensor with the change of copper ion solution concentration is shown in Fig. 3(a). It can be seen that the wavelength of the sensor shifts to long wave with the increase of copper ion solution concentration. Fig. 3(b) shows the wavelength drift of sensors with different length TCF in the concentration range of $0-250 \mu \mathrm{M}$, and in Fig.6(c)(d), the wavelength drift of $0-25 \mu \mathrm{M}$ and $25-250 \mu \mathrm{M}$ are measured respectively. The results show that sensor has a good linear relationship and the sensitivity of the sensor increases with the length of the sensor. In the concentration range of $0-25 \mu \mathrm{M}$, the highest sensitivity of the sensor is $0.0573 \mathrm{~nm} / \mu \mathrm{M}$, and the linearity is $95.33 \%$. In the concentration range of $25-250 \mu \mathrm{M}$, the highest sensitivity of the sensor is $0.0023 \mathrm{~nm} / \mu \mathrm{M}$, $92.96 \%$. In this experiment, the maximum detection limit (LOD) of the sensor is $0.8726 \mu \mathrm{M}$, which indicates that the sensor is sensitive enough to evaluate the concentration of copper ion in water. 

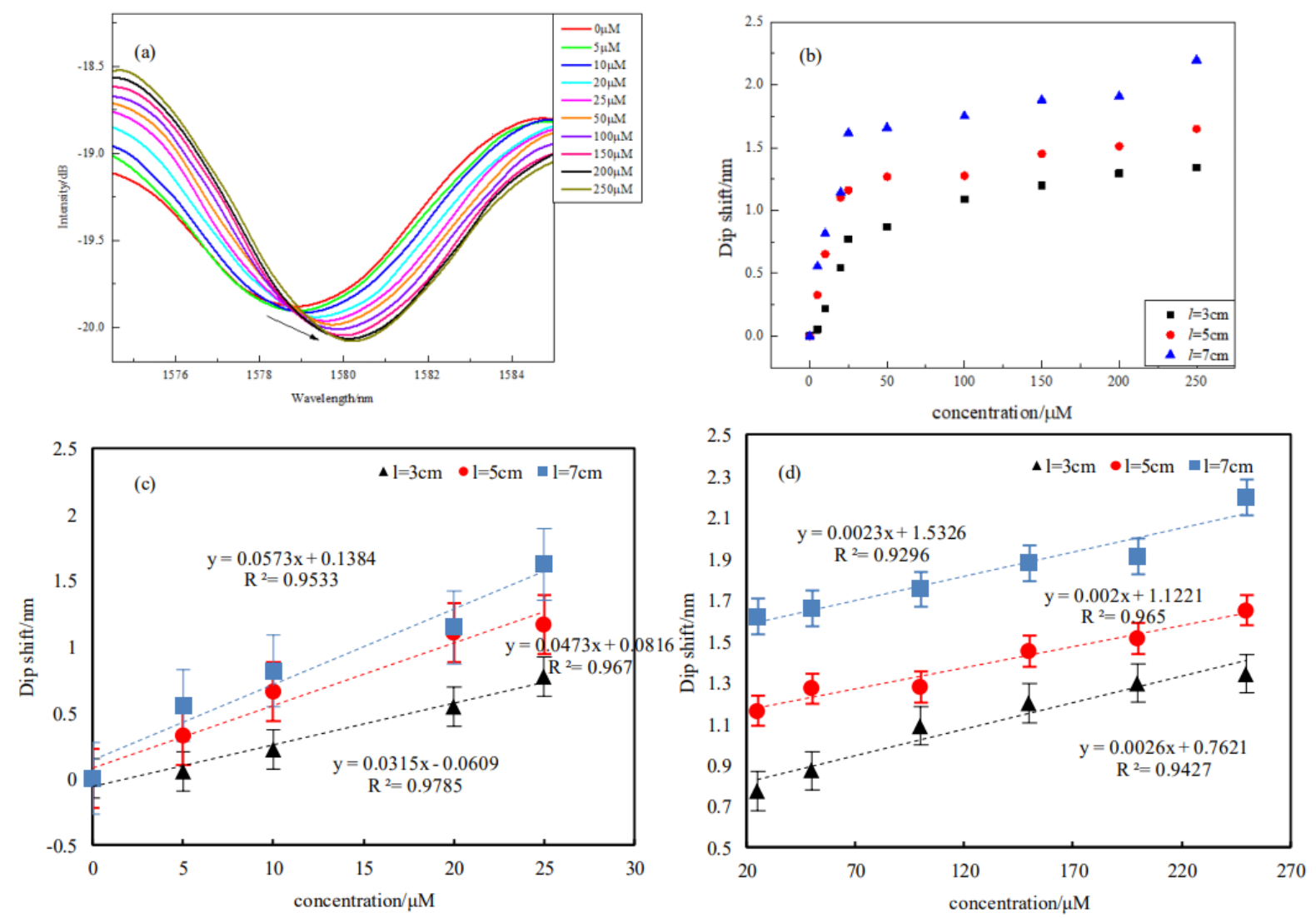

Fig. 3 Response diagram of different length sensors to wavelength change (a) wavelength superposition diagram of STS1 sensor with copper ion concentration at $1578.8598 \mathrm{~nm}(1=3 \mathrm{~cm})(\mathrm{b})$ Wavelength drift diagram of different sensors (c) Fitting diagram of wavelength variation of sensor in the concentration range of $0-25 \mu \mathrm{M}$ (d) Fitting diagram of wavelength variation of sensor in the concentration range of $25-250 \mu \mathrm{M}$

\section{Conclusion}

In conclusion, this paper proposes a MZ interference sensor for the detection of copper ions in aqueous environment, and carries out experimental research. A new type of pyridine compound with triple bond bridge is used to functionalize the sensor for the high sensitivity detection of copper ions. In the range of $0 \mu \mathrm{M}-25 \mu \mathrm{M}$ copper ion concentration measurement, the wavelength changes linearly, and the sensitivity can reach $0.0573 \mathrm{~nm} / \mu \mathrm{M}$, and the detection limit is $0.8726 \mu \mathrm{M}$. The sensor has the advantages of low cost, fast response, compact structure, wide measurement range, good detection effect and application prospect, and can replace the traditional heavy metal ion detection method.

\section{References}

1. Cesar G, Fraga. Relevance, essentiality and toxicity of trace elements in human health.[J].Molecular aspects of medicine.2005: 235-244.

2. Janet Y, Uriu-Adams, Carl L. Keen. Copper, oxidative stress, and human health[J]. Molecular Aspects of Medicine,2005,26(4-5).

3. Li Z L, Song L H. Interpretation of the Guidelines for drinking-water quality(fourth edition) issued by World Health Organization.[J].Water \&
Wastewater Engineering,2012,48(07): 9-13

4. A.E. Sorenson,P.M. Schaeffer. A new bivalent fluorescent fusion protein for differential $\mathrm{Cu}(\mathrm{II})$ and $\mathrm{Zn}(\mathrm{II})$ ion detection in aqueous solution[J]. Analytica Chimica Acta,2020,1101.

5. Mohammadi S Z,Shamspur T,Baghelani Y. Mohammad. Determination of copper, nickel, manganese and cadmium ions in aqueous samples by flame atomic absorption spectrometry after simultaneous coprecipitation with $\mathrm{Co}(\mathrm{OH})_{2}[\mathrm{~J}]$. Arabian Journal of Chemistry,2019,12(7).

6. Lorena C. Martiniano,Vivia R. Abrantes,Sakae Y. Neto, et al. Direct simultaneous determination of $\mathrm{Pb}$ (II) and $\mathrm{Cu}(\mathrm{II})$ in biodiesel by anodic stripping voltammetry at a mercury-film electrode using microemulsions[J]. Fuel,2013,103.

7. Zeng $\mathrm{H} \mathrm{H}$,Thompson Richard B,Maliwal Badri P, et al. Real-time determination of picomolar free $\mathrm{Cu}$ (II) in seawater using a fluorescence-based fiber optic biosensor.[J]. Analytical chemistry,2003,75(24).

8. Lijun Li,Qian Ma,Maoyong Cao,Guina Zhang,Yan Zhang,Lu Jiang,Chunting Gao,Jia Yao,Shunshun Gong,Wenxian Li. High stability Michelson refractometer based on an in-fiber interferometer followed with a Faraday rotator mirror[J]. Sensors \&amp; Actuators: B. Chemical,2016,234. 
9. X.J. Wang peng, Zhao Jingjing, Wang Zhongwei, Cao Delong, Synthesis and Structure Research of a Novel Tribond Bridged Bipyridine Complex, Chinese J. Org. Chem. 31 (2011) 757-761. 